\title{
wissen kompakt „Kieferorthopädie“
}

\begin{abstract}
Insbesondere in der Kieferorthopädie führen ein enormer Wissenszuwachs und stetig neue technische Entwicklungen zu Erweiterungen der diagnostischen sowie therapeutischen Möglichkeiten. Und dennoch stellen auch heute traditionelle Behandlungskonzepte weiterhin bewährte und erfolgreiche Therapieoptionen dar. Die September-Ausgabe von wissen kompakt beleuchtet daher das umfangreiche Gebiet der Kieferorthopädie, setzt besondere Schwerpunkte und widmet sich spannenden, nicht alltäglichen Beiträgen in kompakter Form aufbereitet.
\end{abstract}

\section{Gingivainvaginationen bei kieferorthopädischem Lückenschluss} PD Dr. Kristina Bertl, PhD MSc | Malmö, Wien

Gingivainvaginationen treten mit hoher Prävalenz beim kieferorthopädischen Lückenschluss nach Zahnextraktion auf. Die Ätiologie ist noch ungeklärt. Relevante Folgen sind ein verzögerter und inkompletter Lückenschluss. Der Fortbildungsbeitrag fokussiert sich auf Prävalenz und Folgen von Gingivainvaginationen und verschafft Ihnen einen Überblick zu ÄtiologieTheorien und verschiedenen Therapiemöglichkeiten.

\section{Pathologische Kiefergelenksbefunde im Kindes- und Jugendalter - Häufigkeit, Ursachen und Therapiemöglichkeiten \\ Dr. Daniel Heekeren \\ Dr. Alexandra Lange \\ Prof. Dr. Britta A. Jung | Freiburg \\ Kraniomandibuläre Dysfunktion im Kindes- und Jugendalter ist nicht selten und ungewöhnlich, allerdings ist die Symptoma- tik mehrheitlich weniger ausgeprägt, so dass pathologische Kie- fergelenksbefunde meist erst zu einem späteren Zeitpunkt bei akuter Schmerzsymptomatik entdeckt werden. Das Hauptziel des Beitrags ist es daher, für die Thematik zu sensibilisieren und diagnostische Verfahren, Therapiemöglichkeiten sowie eine sys- tematische CMD-Diagnostik vorzustellen.}

\section{Lückenschluss durch kieferorthopädische Verfahren - wann und bei wem ist er sinnvoll? \\ Prof. Dr. Britta A. Jung \\ Prof. Dr. Dr. Martin Kunkel | Freiburg, Bochum \\ Die therapeutischen Möglichkeiten zur Versorgung von Zahn- lücken sind vielfältig - sie reichen von Autotransplantation über prothetische bis hin zu implantologischen Optionen. Durch die stetige Weiterentwicklung bietet die Kieferorthopädie mittler- weile die Möglichkeit auch in Grenzsituationen kieferorthopä- disch Lücken zu schließen. Der Beitrag widmet sich daher den Vorteilen und der Prognose von kieferorthopädischen Lücken- schlussverfahren und beleuchtet $u$. a. auch die Prognosen von endodontisch versorgten und autotransplantierten Zähnen.}

\section{Obstruktive Schlafapnoe bei Kindern und Jugendlichen} Prof. Dr. Dr. Edmund Rose | Dietikon, $\mathrm{CH}$

Obstruktive Schlafapnoe stellt bei Kindern und Jugendlichen eine eigenständige Diagnose dar, die sich klinisch und therapeutisch von der Erwachsener unterscheidet. Apnoen bei Kindern bleiben oft eine lange Zeit unerkannt. Die Therapie muss interdisziplinär mit dem Kinderarzt erfolgen. Der vorliegende Beitrag beleuchtet das Thema umfassend und legt einen Fokus auf die diagnostische Einschätzung sowie auf aktuelle kieferorthopädische Therapiemaßnahmen.

Die aktuelle Online-Ausgabe von wissen kompakt finden Sie unter www.springerzahnmedizin.de/wissen-kompakt

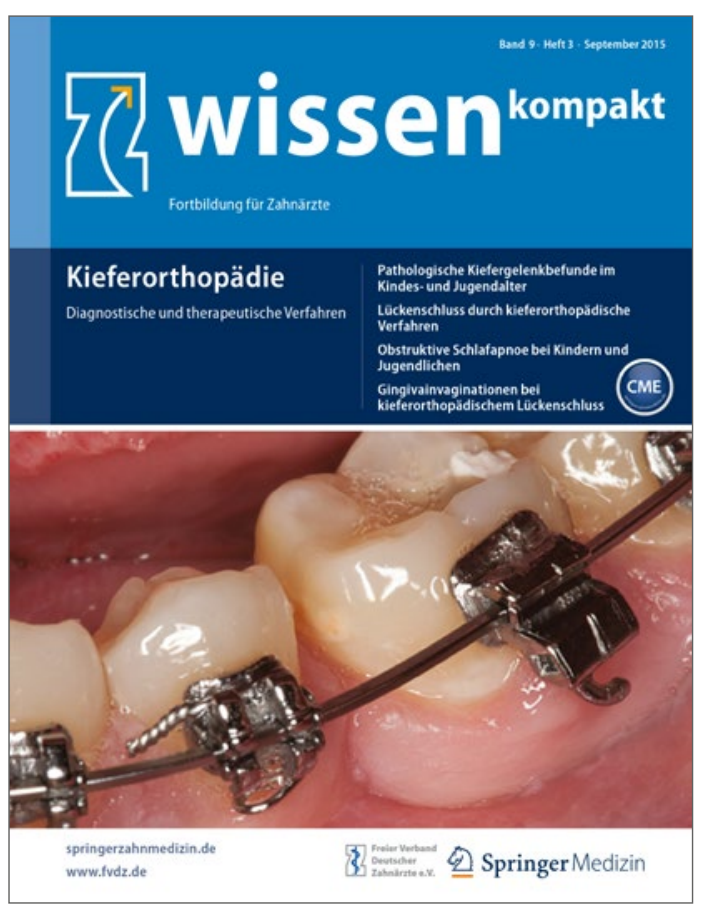

